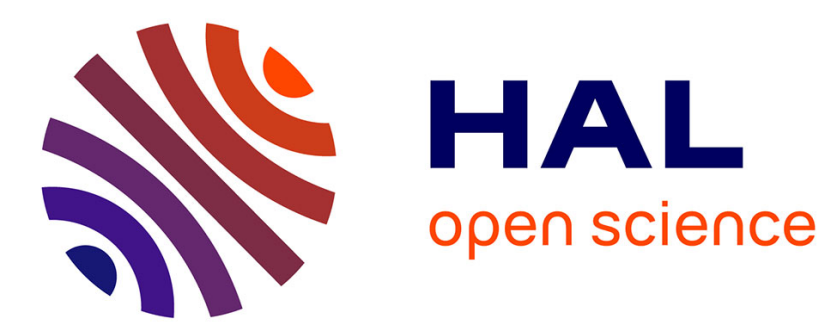

\title{
Regards croisés sur les objets et les pratiques scientifiques et techniques
}

Stéphanie Lacour, Olivier Leclerc, Laurence Dumoulin

\section{To cite this version:}

Stéphanie Lacour, Olivier Leclerc, Laurence Dumoulin. Regards croisés sur les objets et les pratiques scientifiques et techniques. Cahiers, Droit, Sciences \& Technologies, 6, pp.11-163, 2016, $10.4000 /$ cdst.517 . halshs-01410322

\section{HAL Id: halshs-01410322 \\ https://shs.hal.science/halshs-01410322}

Submitted on 1 Feb 2021

HAL is a multi-disciplinary open access archive for the deposit and dissemination of scientific research documents, whether they are published or not. The documents may come from teaching and research institutions in France or abroad, or from public or private research centers.
L'archive ouverte pluridisciplinaire HAL, est destinée au dépôt et à la diffusion de documents scientifiques de niveau recherche, publiés ou non, émanant des établissements d'enseignement et de recherche français ou étrangers, des laboratoires publics ou privés. 


\title{
Regards croisés sur les objets et les pratiques scientifiques et techniques
}

\author{
par Stéphanie Lacour, Directrice de recherche au CNRS, ISP, ENS Cachan, CNRS, \\ Université Paris-Saclay \\ Olivier Leclerc, Chargé de recherche au CNRS, CERCRID (UMR 5137), Université de \\ Lyon, Université Jean Monnet, \\ et Laurence Dumoulin, Chargée de recherche au CNRS, PACTE (UMR5194), \\ Université Grenoble Alpes
}

Référence : Stéphanie Lacour, Olivier Leclerc et Laurence Dumoulin, "Regards croisés sur les objets et les pratiques scientifiques et techniques ", Cahiers Droit, Sciences \& Technologies, $\mathrm{n}^{\circ}$ 6, 2016, pp. 11-22.

DOI : https://doi.org/10.4000/cdst.517

\begin{abstract}
« Ceux qui se bornent à une seule recherche manquent souvent de faire des découvertes qu'un esprit plus étendu, qui peut joindre d'autres sciences à celles dont il s'agit, découvre sans peine. Mais comme un seul ne saurait bien travailler à tout, c'est l'intelligence mutuelle qui peut y suppléer. " Nouvelles lettres et opuscules inédits de Leibniz, rassemblés par L.A. FOUCHER DE CAREIL, Paris, 1857, p. 287.
\end{abstract}

Parce que l'intelligence des dispositifs sociotechniques, de ce qu'ils sont comme de ce qu'ils produisent, s'enrichit de regards pluridisciplinaires, parce que les échanges entre le droit et les autres sciences humaines et sociales sont finalement rares, ce dossier, issu d'une journée d'études qui s'est tenue en décembre 2014 à Cachan, sous l'égide du réseau Droit, Sciences et Techniques et de l'Institut des Sciences Sociales du Politique, propose de croiser ces perspectives à propos d'objets et de pratiques scientifiques et techniques. Au-delà des thèmes explorés dans chacune des contributions, ce sont les notions et les pratiques de l'interdisciplinarité, les dynamiques que celles-ci supposent tout comme les déplacements théoriques et épistémologiques qu'elles engendrent, que ces articles éclairent.Le caractère polysémique de la notion d'interdisciplinarité a maintes fois été souligné. Parfois, il s’est agi de regretter un usage abusif ou exagérément extensif ${ }^{1}$. Souvent, c'est pour tenter de mieux le saisir qu'on a

1 Ainsi, regrettant qu'elle devienne parfois un "slogan utilisé à tort et à travers », V. G. GUSDORF, "Passé, présent, avenir de la recherche interdisciplinaire ", in UNESCO, Interdisciplinarité et sciences humaines, Paris, Unesco, 1983, p. 31 et s., spéc. p. 38. De même sur la pluridisciplinarité prétendue des rencontres organisées entre droit public et droit privé, qualifiée de « facilité de langage 
décliné la litanie des préfixes apposés à la discipline comme autant de variations sur un même thème. «Inter » - «pluri » - «trans » - « multi » - disciplinarités, la liste fluctue. Toutes ces distinctions ont été balisées ${ }^{2}$ à l'envi sans, faut-il le noter, qu'un accord général n’ait pour l'instant été trouvé quant à leurs territoires respectifs. Nous nous contenterons, dans la suite de notre propos, de parler d'interdisciplinarité, pour remarquer que, inter ou pas, c'est avant tout de disciplinarité qu'il s'agit. En effet, l'interdisciplinarité est indissociable de la structuration et de l'institutionnalisation des savoirs en disciplines ${ }^{3}$.

Chacun sait bien que les contours d'une discipline, tant sur le terrain de ses objets, de ses méthodes, des rapports de sociabilité savante qu'elle abrite, ne sont jamais donnés: les frontières dressées sont toujours discutées, contestées, activement maintenues, sans cesse recomposées ${ }^{4}$. Penser l'interdisciplinarité dans la recherche juridique ne peut donc faire l'économie d'une réflexion sur la manière dont le droit comme discipline a établi un espace intellectuel propre ${ }^{5}$. Cette histoire, maintes fois contée, est celle d'une lutte pour le maintien et la conquête d'un espace légitime, que ce soit à l'encontre de la sociologie naissante ${ }^{6}$, de l'histoire ${ }^{7}$, ou plus largement des disciplines des sciences humaines et sociales ${ }^{8}$.

Dès lors s'engager dans une démarche interdisciplinaire c'est d'abord renouer ce qui a été dénoué, dessiner des transversales à travers des frontières patiemment érigées, reproduites via des pratiques d'enseignement et de socialisation et soutenues par de puissants renforts institutionnels.

qui interdit de s’interroger sur l'utilité de construire un objet pluridisciplinaire », L. ZÉVOUNOU, Introduction à l’ouvrage " Usages de l'interdisciplinarité », sous la direction de E. BOTTINI et al., Presse universitaires de Paris Ouest, 2014, p. 11 et s., spéc. p. 13.

2 S. N. SMIRNOV, «L'approche interdisciplinaire dans la science aujourd'hui : fondements ontologiques et épistémologiques, formes et fonctions », in UNESCO, op. cit. p. 53 et s. Voir aussi, F. OsT et M. VAN DE KERCHOVE, « De la scène au balcon, d'où vient la science du Droit », in Normes juridiques et régulation sociale, sous la direction de F. Chazel et J. Commaille, Paris LGDJ, « Droit et Société », 1991, p. 77 et s.

3 Y. GingRAS, "L'institutionnalisation de la recherche en milieu universitaire et ses effets », Sociologie et sociétés, vol. 23, nº 1, 1991, p. 41-54.

4 Voir par exemple N. Defaud et V. GuIADER, Discipliner les sciences sociales. Les usages sociaux des frontières scientifiques, Paris, L'Harmattan, 2002.

5 D. W. VICK, « Interdisciplinarity and the Discipline of Law », in Journal of Law and Society, vol. 31, $\mathrm{n}^{\mathrm{O}}$ 2, 2004, p. 163-193.

6 A.-J. Arnaud, Les juristes face à la société du XIXe siècle à nos jours, Paris, Puf, 1975 ; F. SOUBIRAN-PAILlET, «Juristes et sociologues français d'après-guerre: une rencontre sans lendemain », Genèses, no 4, 2000, p. 125-142 ; F. AUDREN et J.-L. HALPERIN, La culture juridique française. Entre mythes et réalités $X I X^{e}-X X^{e}$ siècles, Paris, CNRS Éditions, 2013.

7 F. SOUBIRAN-PAILlET, « Histoire du droit et sociologie : interrogations sur un vide disciplinaire », Genèses, 29, 1997, p. 141-163 ; J.-L. HALPERIN, « L’histoire du droit constituée en discipline : consécration ou repli identitaire ? », Revue d'histoire des sciences humaines, vol. 1, nº 4, 2001, p. 932.

8 Voir les contributions réunies dans l’ouvrage collectif placé sous la direction de Philip A. THOMAS qui envisagent les rapports du droit et de nombreuses disciplines comme la psychologie, la philosophie, l'économie, la géographie, etc. : Philip A. THOMAS, Legal Frontiers, Aldershot, Dartmouth, 1996. 
En outre, les frontières de la discipline juridique, et les rapports qu'elle entretient avec les domaines du savoir proches ou lointains, ne sont pas nécessairement configurés de la même manière en France et à l'étranger. Par conséquent, entreprendre un travail interdisciplinaire, ce n'est pas seulement engager un parcours dans un espace intellectuel marqué par une histoire longue, c'est aussi se situer dans des constructions nationales et internationales qui présentent, selon les disciplines, des caractères propres. Ainsi par exemple, pour l'étude des activités de justice, les croisements entre droit et sociologie du droit ne s'opèrent pas à l'identique en France et au Royaume-Uni ${ }^{9}$. En France, la "sociologie juridique » a bien souvent placé la sociologie dans une position ancillaire quand les socio-legal studies sont aujourd'hui largement diffusées dans les facultés de droit au Royaume-Uni, au point qu'un auteur n’hésite pas à affirmer, non sans une pointe d'exagération sans doute, que «nous sommes tous socio-legal maintenant $»^{10}$ ! Dès lors, une recherche qui, là, paraîtra interdisciplinaire, sera ailleurs inscrite au sein d'une discipline, dont les contours peuvent différer. Les conséquences ne sont pas seulement intellectuelles ; elles sont aussi institutionnelles. L'évaluation de la recherche se faisant le plus souvent au sein des disciplines, le meilleur moyen de faire une carrière avec des travaux interdisciplinaires peut être d'inscrire son travail dans un espace intellectuel où ces travaux sont disciplinaires $!^{11}$.

Si notre conception de l'interdisciplinarité s'inscrit, par hypothèse, dans l'espace intellectuel que dessinent les disciplines et à l'intérieur de leurs cadres, elle promeut leur croisement, leur enrichissement mutuel mais surtout l'exploration des « confins ", « ces bandes de territoire jouxtant les frontières d'une discipline d'où, tout en restant dans la sécurité de leur propre discipline, les chercheurs peuvent s'interpeller et communiquer avec des scientifiques de disciplines différentes qui eux-mêmes demeurent aux confins de leur territoire disciplinaire ${ }^{12}$. L'interdisciplinarité est un jeu de croisements, que l'on espère productif ${ }^{13}$, entre différentes disciplines. Des disciplines dont on souhaite tour à tour compléter, affiner, rénover, combiner ou encore mieux observer et comprendre les méthodes, les résultats et les pratiques grâce aux apports intellectuels venant d'autres domaines du savoir.

9 E. Serverin, Sociologie du droit, Paris, La Découverte, 2000 ; Th. DelPeuch, L. Dumoulin, C. de Galembert, Sociologie du droit et de la justice, Paris, Armand Colin, 2014.

10 C. McCrudDEN, « Legal Research and the Social Sciences », Law Quarterly Review, vol. 122, 2006, p. 645.

11 Cela n'est pas sans lien avec ce qui peut apparaître, à première vue, comme un paradoxe : alors que l'interdisciplinarité constitue une tentative de réponse aux limites cognitives et institutionnelles des disciplines et un moyen de contourner des formes de contrôle et de normativité parfois perçues comme restrictives, elle évolue la plupart du temps vers des structurations reproduisant les schémas disciplinaires.

12 A. MARCOVich et T. SHINN, «Quelle disciplinarité pour la recherche à l’échelle nano ? La « nouvelle disciplinarité », aux « confins » des disciplines », Actes de la recherche en sciences sociales, vol. 210, $\mathrm{n}^{\mathrm{O}}$ 5, 2015, p. 50-59.

13 Voir, par exemple, pour une explicitation des apports d'une analyse croisée à partir du droit et des sciences économiques : A. JEAMMAUD, «L’interdisciplinarité, épreuve et stimulant pour une théorie des règles juridiques ", in Le droit dans l'action économique (dir. T. KIRAT et É. SERVERIN), Éditions du CNRS, 2000, p. 219-231. 
Croiser les savoirs disciplinaires n’est donc pas nécessairement contester les disciplines en tant que telles ni concourir à leur dissolution. Au contraire, dans le débat nourri qui s'est développé autour de l'interdisciplinarité - non seulement entre sciences sociales, mais aussi entre sciences sociales et sciences de la nature -, certains auteurs réaffirment l'utilité et la nécessité des disciplines, en tant qu'elles structurent et stabilisent les conditions de production du savoir scientifique et de sa cumulativité ${ }^{14}$. De cette inscription disciplinaire et de la stabilité qu'elle offre, peut naître une interdisciplinarité, entendue non comme une fin en soi mais comme une forme d'ouverture, de "culture du dépaysement ${ }^{15}$ - une des voies disponibles pour faire avancer l’intelligence des phénomènes analysés ${ }^{16}$.

Sauter le pas de l'interdisciplinarité, c'est ainsi faire le pari que sur un plan intellectuel, les frontières des disciplines sont aussi productives que contreproductives : elles rendent possible la production d'un certain savoir, et dans le même temps elles rendent d'autres savoirs invisibles, inaccessibles. L'interdisciplinarité est de fait souvent une réponse aux limites de chaque discipline, limites identifiées par certains de leurs membres $^{17}$. L'interdisciplinarité revient à chercher ce qui peut être trouvé dans les espaces que les disciplines ont rendu inféconds. Partant, l'interdisciplinarité s'opère toujours depuis une discipline, exactement comme le droit comparé se fait à partir d'un questionnement né dans un système juridique donné : une recherche juridique interdisciplinaire n'apporte pas la même chose qu'une recherche sociologique, historique, économique interdisciplinaire. L'une et l'autre peuvent être fécondes, mais elles ne comblent pas les mêmes angles morts. L'interdisciplinarité suppose donc, en définitive, une traduction: qu'est-ce que les autres disciplines peuvent apporter d'intéressant? La réponse à cette dernière question est nécessairement disciplinaire ${ }^{18}$. Ainsi, on ne pourra pas ignorer à la lecture de ce dossier qu'il est le fruit d'une initiative provenant principalement de juristes.

S'il n’y a pas d' « ailleurs », pas de questionnement qui ne plonge ses racines dans une discipline, il y a une multitude de chemins, mille manières de croiser et d'enrichir les rapports entre les disciplines ${ }^{19}$. Le droit peut-il se prêter à ce dialogue ?

14 Par exemple J. JACOBS, In Defense of Disciplines. Interdisciplinarity and Specialization in the Research University, Chicago, Chicago University Press, 2014.

15 I. Stengers, Cosmopolitiques, t. 1, La guerre des sciences, Paris, La Découverte/Les empêcheurs de penser en rond, 1996, p. 100-101 et 110 reproduit dans Hermès, La Revue, vol. 67, nº 3, 2013, p. 201.

16 J. Heilbron et Y. Gingras, "La résilience des disciplines », Actes de la recherche en sciences sociales, vol. 210, $\mathrm{n}^{\mathrm{0}}$ 5, 2015, p. 4-9.

17 J. Commaille, "Les nouveaux enjeux épistémologiques de la mise en contexte du droit », Revue interdisciplinaire d'études juridiques, vol. 70, nº 1, 2013, p. 62-69.

18 Ce dont témoigne par exemple la structure des deux dossiers consacrés par la revue Droit et société à la réflexion interdisciplinaire sur le droit : dossier «Quelles méthodes pour la sociologie du droit et de la justice ? », vol. 69-70, $\mathrm{n}^{0}$ 2-3, 2008 ; et son pendant en direction des juristes « Sciences sociales, droit et science du droit : le regard des juristes », vol. 75, nº 2, 2010, p. 267-273.

19 Voir les parcours de recherche décrits in V. LEMAY et F. DARBELLAY (dir.), L'interdisciplinarité racontée. Chercher hors frontières, vivre l'interculturalité, Peter Lang, 2014. Voir également les différentes illustrations données dans « Interdisciplinarité : entre disciplines et indiscipline », numéro spécial, Hermès, La Revue, vol. 67, nº 3, 2013. 
Certains en doutent. Ainsi, pour Geoffrey Samuel, le savoir juridique s'est érigé comme discipline autour d'un paradigme de l'autorité qui consiste en une mise en ordre autoréférencée d'énoncés signifiant des normes. Ne se donnant pour objet aucun phénomène empirique, la discipline juridique n'aurait dès lors guère d'intérêt pour les autres disciplines, ce qui rendrait l'échange interdisciplinaire bien peu fructueux, si ce n'est tout simplement illusoire ${ }^{20}$.

Les contributions réunies dans ce dossier se prêtent à une appréciation plus optimiste. Elles illustrent, chacune à leur manière, le fait que l'interdisciplinarité ne se conforme pas à un mode d'emploi unique : pas plus qu'il n’y a qu'une seule manière de « faire du droit ${ }^{21}$, il n'y a pas une seule manière d'entreprendre un travail interdisciplinaire. C'est par la diversité de ses pratiques que ce dossier entend éclairer l'interdisciplinarité. En témoignent la variété des motifs du recours à des approches interdisciplinaires, la forme et le périmètre qu'elles épousent, les résultats qu'elles permettent d'obtenir.

\section{1. Éclairer l’interdisciplinarité par trois questions}

1.1. Les raisons du recours à des approches interdisciplinaires sont tout à la fois personnelles à chaque chercheuse et à chaque chercheur et liées au positionnement des institutions et des structures dans lesquelles ils exercent leur métier.

Du point de vue individuel, plusieurs types de motifs conduisent des chercheurs à se tourner vers des approches interdisciplinaires.

Leurs motivations peuvent tout d'abord être d’ordre épistémique. Tel sera le cas lorsque l'analyse disciplinaire, même poussée à son paroxysme, laisse le chercheur insatisfait, sur sa faim, quant aux résultats qu'il parvient à produire, dans le cadre de sa spécialisation académique. De même, les méthodologies disponibles dans un champ disciplinaire peuvent parfois s'avérer insuffisantes aux yeux de chercheurs, confrontés à un objet spécifique. Enfin, il peut arriver qu'un objet de recherche pris en considération par d'autres domaines de la connaissance fasse son apparition dans une nouvelle discipline. Ces raisons constituent autant d'incitations à se tourner vers d'autres sources de réflexions.

Les motivations des chercheurs peuvent également être d'ordre stratégique. Tel sera le cas, par exemple, s’ils estiment que la structuration de groupes interdisciplinaires permet la fabrication de "nouveaux» espaces de légitimité, débouchant sur de nouveaux canaux de reconnaissance, et dégageant potentiellement des perspectives accrues de moyens et de postes... Cette mobilisation stratégique de l'interdisciplinarité peut d'ailleurs, dans de telles circonstances, s'avérer conjoncturelle, par exemple

20 G. SAMUEL, «Interdisciplinarity and the Authority Paradigm: Should Law Be Taken Seriously by Scientists and Social Scientists? », Journal of Law and Society, vol. 36, nº 4, 2009, p. 431-459.

21 A. JEAmmaUd, «La part de la recherche dans l'enseignement du droit », Jurisprudence. Revue critique, $\mathrm{n}^{0} 1,2010$, p. 181. 
lorsqu'un financement ad hoc est attaché à une approche interdisciplinaire, dans le cadre de politiques scientifiques incitatives déployées à différentes échelles - régionale, nationale ou internationale.

D'un point de vue plus collectif, cette fois, les motifs peuvent être alternativement de type top-down ou bottom-up.

Dans le premier cas, une demande de politique scientifique émanera d'institutions variées - laboratoires, groupes de recherche, organismes ou universités, ministères, fondations privées ou publiques, institutions internationales, etc. - visant à mettre en œuvre des recherches interdisciplinaires. Cette demande, si elle est récurrente, peut être exprimée avec plus ou moins de conviction. Elle est, suivant les périodes et les institutions, arrimée à différents types d'objectifs et inégalement appuyée sur des dispositifs concrets de facilitation de l'interdisciplinarité. La question des financements et des moyens matériels mis à la disposition des chercheurs et d'un certain type de recherche est d'ailleurs loin d'être secondaire. Il est aisé de constater que différents mouvements intellectuels autour du droit, comme Law \& Society et Law \& Economy, par exemple, se sont constitués en s’appuyant sur certains financeurs spécifiques ${ }^{22}$.

Dans le second cas, le recours à l’interdisciplinarité est perçu comme nécessaire au vu de l'actualité sociale et politique dans laquelle les chercheurs sont immergés, y compris en dehors de leur activité professionnelle. La sensibilité des chercheurs, quand ce n'est pas leur participation, à des mouvements sociaux qui dépassent le monde de la recherche favorise des questionnements qui ne restent pas prisonniers des disciplines : la cause des minorités, des femmes, la lutte contre les discriminations... Ce type d'influence est peut-être moins pérenne et la stabilité des champs de recherche interdisciplinaires qui en découlent dépendra, de fait, assez étroitement, du sort des causes ainsi conceptualisées dans l'espace public : les études sur la sécurité, qu'elle soit globale ou pas, semblent ainsi promises à un bel avenir, pour ne donner qu'un seul exemple.

Bien entendu, ces deux niveaux (individuel et collectif) ne sont distingués ici qu'à des fins de clarification du propos et des idées, de même que les motifs dégagés sont rarement présents à l'état pur, ou stables dans le temps. Un même chercheur peut d'abord s'engager dans un projet disciplinaire pour répondre à une commande voire à une injonction, puis y trouver un "supplément d’âme " (intérêt intellectuel, qualité d'échanges, rencontres et affinités électives avec d'autres chercheurs, formes de socialisation académiques différentes de celles qu'il connaît) qui motivera la poursuite du projet. Ou... inversement !

1.2. Les différentes formes du travail interdisciplinaire. Tout comme sont variées les raisons qui peuvent expliquer qu'un chercheur ou un groupe de chercheurs ait recours à des approches interdisciplinaires, les formes que ces dernières peuvent prendre sont multiples. Celles-ci vont de simples échanges ponctuels à la constitution de

22 A. VAuchez, «Entre droit et sciences sociales. Retour sur l'histoire du mouvement Law and Society », Genèses, Vol 4, nº 45, 2001, p. 134-149. 
groupes de recherche pérennes; de la juxtaposition de points de vue disciplinaires différents sur un ou des objets communs à la production de nouveaux objets et méthodes plus ou moins émancipés des disciplines instituées ; de la mise en perspective critique des savoirs et méthodes de chacun à l'élaboration d'une perspective nouvelle qui leur soit commune.

Il faut toutefois noter que si elle peut prendre la forme de réflexions individuelles et d'incursions ponctuelles dans des disciplines connexes, la recherche interdisciplinaire repose souvent sur la création de groupes ou de communautés transversales développant des approches collectives menant, dans le meilleur des cas, à la constitution de corpus et de méthodes nouveaux.

Le choix d'une manière de faire reflète plus profondément un engagement d’ordre épistémologique. Deux grandes conceptions peuvent être mises en contraste, même si elles sont loin d'être toujours explicites.

D’un côté, une interdisciplinarité conçue comme un dialogue (plus ou moins étroit, plus ou moins constructif) entre des chercheurs issus de disciplines distinctes, insérés dans des communautés disciplinaires propres, maîtrisant des corpus, des méthodes, des manières différentes d'interroger le monde. L’interdisciplinarité est ici vue comme une manière de travailler ensemble, malgré les différences, de rapprocher, le temps de projets communs, des individus formés et exerçant leur recherche au sein de disciplines distinctes.

Une deuxième conception de l'interdisciplinarité correspond à un processus de construction d'une intersection au carrefour de plusieurs disciplines, souvent rendue cohérente, du moins en théorie, par une communauté d'objets - typiquement les studies - définissant des méthodes originales, des notions nouvelles, et, idéalement, une communauté scientifique s’autonomisant par rapport aux disciplines " classiques ». En somme, cette deuxième vision de l'interdisciplinarité désigne des dynamiques de construction de disciplines nouvelles (d'interdisciplines?). Encore ne faut-il pas négliger, tout en admettant la force de ces dynamiques, l'ensemble des controverses auxquelles elles sont, en permanence, confrontées ${ }^{23}$.

1.3. Les résultats de la recherche interdisciplinaire. Enfin, il faut le souligner pour terminer ce panorama, la recherche interdisciplinaire aboutit à des résultats tout aussi protéiformes. Elle peut mener à un échec pur et simple, parfois cuisant, partant du constat d'une incompréhension mutuelle ou d'une absence totale d'intérêt des tiers (et en particulier des communautés disciplinaires d'origine) pour la démarche adoptée et pour ses résultats. Elle peut aussi être une véritable réussite à l’intérieur du groupe ayant

23 Les débats houleux qui ont opposé, en 1999, Steve Fuller et Joan H. Fujimura dans les colonnes de l'American Anthropologist ne sont que l'un des épisodes de ces controverses dans les Science and Technology Studies, qu'il faut peut-être lire comme le signe positif d'une permanence des débats dans la communauté des chercheurs impliqués dans ce champ d'études. V. S. Fuller, “Authorizing Science Studies: Or, Why we have never had paradigms”, American Anthropologist, vol. 101, $\mathrm{n}^{0} 2$, June 1999, p. 379-381 et J.H. Fujimura, “Authorizing Science Studies”, American Anthropologist, vol. 101, $n^{0} 2$, June 1999, p. 381-384. 
lancé l'initiative interdisciplinaire, mais ne pas avoir d'écho à l'extérieur, du fait d'initiatives concurrentes ou encore d'un manque de visibilité institutionnelle. Parfois, par un effet en retour, elle sera, sans que cela ait toujours été prévu par des initiateurs, à la source de profonds bouleversements des cadres d'une ou plusieurs des disciplines d'origine, de leurs méthodes, de leurs objets, avec, à la clé, la possible création d'une nouvelle discipline à part entière, dont on formera, à l'avenir, des spécialistes.

À titre d'exemple, dans le domaine des sciences et des techniques, les Science and Technology Studies (STS) ${ }^{24}$ se sont efforcées, depuis les années 1970, de faire converger le regard de différentes disciplines des sciences sociales sur la science telle qu'elle se fait. À émergé ainsi un domaine de connaissance auquel contribuent, le plus souvent de manière indistincte, historiens, philosophes, politistes, sociologues des sciences et techniques. Des juristes sont également engagés dans ce champ de recherche $^{25}$. Ces chercheurs, parfois en revendiquant la double généalogie de leurs inscriptions disciplinaires d'origine et celle du mouvement $\mathrm{STS}^{26}$, ont pratiqué des formes d'interdisciplinarité dont on peut estimer qu'elles ont, au moins partiellement, atteint leur but. Leurs travaux ont en effet contribué à la reconnaissance de ce domaine de recherches et à son institutionnalisation autour d'un ensemble d'outils : notions et concepts clés, éléments méthodologiques, revues, société savante, et même chaires d'enseignement dans certains cas. D'une façon comparable et sensiblement à la même période, le mouvement américain Law and society s'est attaché à faire émerger l'image du droit comme interagissant avec le social. Il a fédéré des contributions disciplinaires multiples, histoire, anthropologie, sociologie... ${ }^{27}$ - élargissant ainsi les coopérations entre droit et sociologie déjà engagées par les réalistes dans les années 1930.

L’interdisciplinarité, par sa relative indétermination théorique, se présente toutefois comme une pratique multiforme, dont l'issue n'est jamais certaine. Dans un paysage académique qui demeure fortement structuré par les disciplines, et malgré des incitations institutionnelles volontaristes, la pratique de l'interdisciplinarité place les chercheurs, au moins initialement, dans une posture de marginalité ${ }^{28}$ et de dépendance, dans leurs inspirations et/ou leurs résultats, à l'égard des disciplines préexistantes.

Entendre l'interdisciplinarité comme un cadre méthodologique fermé, impliquant nécessairement des formes particulières de collaboration ne permet pas de

24 D. Pestre, Introduction aux Science Studies, Paris, La Découverte, 2006 ; Ch. BonneuIL et P.B. Joly, Sciences, techniques et société, Paris, La Découverte, 2013.

25 Voir par exemple G. EDMOND (ed.), Expertise in regulation and law, Aldershot, Ashgate, 2004; Sh. JASANOFF, Le droit et la science en action, textes traduits et présentés par O. LECLERC, Paris, Dalloz, coll. "Rivages du droit», 2013; E. ClOATRE et M. PiCKERSGILL (eds.), Knowledge, Technology and Law, Routledge, 2015.

26 Le terme « mouvement », volontairement plus large et socialement connoté que celui de «studies » est emprunté à Susan Cozzens, qui a été l'éditrice de la revue de référence des STS, «Science, Technology and Human Values » de 1988 à 1993 et insiste sur la question des rapports entre les STS et la justice sociale, en les qualifiant ainsi, V. S. CozZENS, STHV, vol. 18, nº 3, 1993, p. 275.

27 L. Friedman, "The Law and Society Movement », Stanford Law Review, vol. 38, February 1986, p. 763-780; A. Vauchez, op. cit.

28 Ce que soulignent M. DOGAN et R. PAHRE dans L'innovation dans les sciences sociales. La marginalité créatrice, Paris, PUF, 1991. 
rendre compte de manière satisfaisante du foisonnement des expériences interdisciplinaires qui se déploient au carrefour des études des sciences et techniques. Au contraire, en estimant que l'interdisciplinarité doit être pratiquée, mise en œuvre, expérimentée, mais aussi discutée à partir des formes concrètes qu'elle prend et des tentatives, fructueuses ou non qu'elle suscite, nous nous donnons la possibilité d'entrevoir de nouvelles pistes qui nous permettront peut-être, collectivement, de mieux cerner, comprendre et éventuellement expliquer les objets et les pratiques scientifiques et techniques.

\section{Présenter des travaux interdisciplinaires en deux axes}

Les contributions de ce dossier sont ordonnées autour de deux axes. L’interdisciplinarité peut en effet être mobilisée avec profit aussi bien pour comprendre la normativité des sciences et techniques que pour comprendre les recompositions du droit. Ces deux voies ne sont pas les seules possibles. Elles ont leurs limites et, sans doute, des prolongements. Elles sont, en toute hypothèse, complémentaires pour appréhender les recherches interdisciplinaires qui sont menées sur les rapports existants entre le droit, les sciences et les techniques aujourd'hui.

En étudiant les différentes façons dont les sciences et les techniques sont sources de normes, les chercheurs en droit ont croisé les trajectoires d'objets et de pratiques qu'ils ont progressivement cherché à mieux cerner, mesurer, comprendre dans leurs interactions avec les normes juridiques.

Le cas de l’identification par analyse génétique est à cet égard révélateur, selon Bertrand Renard et Inès Gallala ${ }^{29}$, des ambiguïtés qui affectent le recours, pour la recherche de la vérité judiciaire, à des normes issues d'autres sources de véridiction. Ces dernières sont tout à la fois produites par la norme juridique - « l'identification par analyse génétique est une production juridique, au sens où elle n'existe que dans ce que le droit fait de l'ADN » - et partiellement substituées aux équilibres établis en matière de procédure pénale, autrement dit productrices de cette norme, qu'elles importent de manière discrète dans l'arsenal des conventions secondaires qu'il est nécessaire de mieux cerner pour analyser les conditions d'effectivité de lois volontairement simplificatrices. La connaissance de ces conventions secondaires - parmi lesquelles les standards d'analyse, mais aussi les procédures de la banque belge de données génétiques - suppose un accès aux réseaux d'acteurs, notamment scientifiques, qui en déterminent les contenus. C'est à la construction de ces terrains, tout comme à celle des questions scientifiques qu'elles ont spécifiquement formulé au sujet des fichiers d'empreintes génétiques, et plus particulièrement du Fichier National Automatisé des Empreintes Génétiques que se sont, pour leur part, attelées Joëlle Vailly, Florence Bellivier, Christine Noiville et Vololona Rabeharisoa. Leur texte ${ }^{30}$ rend compte des

29 B. RENARD et I. GALlALA, « Le droit, traducteur des enjeux des dispositifs sociotechniques ? Étude de cas à propos de l’identification par analyse génétique en justice pénale », p. 23.

30 J. VAilly, Fl. Bellivier, Chr. Noiville, V. RABEHARISOA, « Les fichiers d'empreintes génétiques et les analyses d’ADN en droit pénal sous le regard du droit et de la sociologie », p. 43. 
choix de méthode et des raisons qui ont guidés leur recherche, axée sur les notions de preuve et d'objet-frontière. L'article fait ressortir tout à la fois l'enrichissement réciproque des questionnements que permet la recherche interdisciplinaire et la forme concrète que prennent toutes les étapes de leurs travaux: entretiens en binômes, nombreuses réunions intermédiaires, et lectures croisées, notamment.

Dans la lignée des conclusions retenues par le texte précédent, l'article rédigé par Volny Fages, Stéphanie Lacour et Sacha Loeve ${ }^{31}$ met l'accent, via une étude interdisciplinaire des nanotechnologies et de l'impression en trois dimensions, sur les dimensions épistémiques du questionnement interdisciplinaire. En mettant en évidence des failles, des décalages entre les trajectoires concrètes et les discours sociaux développés autour des machines qui sont au cœur des deux champs d'innovations qu'ils étudient, et en développant, précisément au creux de ces décalages, une recherche sur la normativité des technosciences, ils justifient l'intérêt d'une approche interdisciplinaire du temps présent des objets qu'ils étudient. Dans le même temps, ils offrent l'illustration de l'intérêt de politiques scientifiques volontaristes de promotion de l’interdisciplinarité. Le projet Jurismart, présenté par Anne-Sophie Denolle, Séverine Nadaud et Thierry Tacheix ${ }^{32}$ suggère, enfin, qu'une telle interdisciplinarité peut également porter ses fruits au-delà même du croisement de savoirs qu'elle permet, et qu'elle possède aussi un caractère opératoire. La production de connaissances au sujet d'objets techniques, les réseaux énergétiques dits intelligents, n'est en effet, selon les termes mêmes des auteurs, pas le seul propos de leur collaboration, qui vise également à identifier et lever les verrous existants au déploiement de ces réseaux.

La normativité des sciences et technologies n'est toutefois que l'un des volets possibles des études interrogeant les relations entre normes, sciences et techniques. Il est également possible de mettre en évidence, par des approches interdisciplinaires, les différentes recompositions dont la norme juridique fait l'objet sous l'influence de développements scientifiques et techniques.

C’est précisément à l'étude d'une norme juridique en cours d'adaptation, le Règlement Novel Food de 1997, qu'est consacré l'article de Gervaise Debucquet et Marine Friant-Perrot ${ }^{33}$. L'étude vise en effet, selon leurs termes, à « croiser le droit et la socio-anthropologie de l'alimentation afin d'apprécier les reconfigurations législatives en cours, leur pertinence, leur incomplétude » et mène, comme ses auteures le montrent bien, à porter sur les catégories forgées par le droit un regard critique qui met en lumière, encore une fois, les discontinuités temporelles dans lesquelles la construction du droit se déploie en réalité. Le concept de nouveauté alimentaire est, à cet égard, un bon révélateur des tensions existant, au sein des catégories forgées par le droit, entre des approches technoscientifiques et des définitions socio-anthropologiques. L'un des intérêts, ici, de l'approche interdisciplinaire adoptée par les auteures est de donner à voir non seulement le droit tel qu'il se présente dans les livres mais aussi tel qu'il s’applique

31 V. FAgES, S. LACOUR et S. LOEVE, « Trajectoires comparées des nanotechnologies et de l'impression 3D. Temporalité et normativité des sciences et technologies », p. 55.

32 A.S. Denolle, S. NAdAud, T. TACheiX, « Le projet Jurismart : une recherche interdisciplinaire sur les réseaux énergétiques intelligents », p. 75.

33 G. Debucquet, M. Friant-Perrot, « Regards croisés sur la notion de Novel Food : quel droit pour quelle nouveauté », p. 81. 
concrètement ${ }^{34}$, dans des espaces et des temporalités variables. Tout comme le travail de Louise Danguy et Nicolas Fortané ${ }^{35}$, qui étudient « la façon dont les liens entre droit et sciences conditionnent l'élaboration des normes ", la construction du droit n'est donc pas appréhendée ici comme une entreprise finalisée, puisqu'une fois les textes édictés, leur " portée normative » évolue en permanence. C’est précisément au cœur de ces évolutions que se situe la clé des analyses portant sur les écologies normatives complexes que mettent en avant ces derniers auteurs pour renouveler l'analyse des relations entre les décideurs et les experts, entre le droit et la science.

Le dossier se clôt par deux contributions portant sur les questions environnementales. M. Bary et M.-H. Hubert envisagent les instruments de régulation de la pollution de l'air et de l'atmosphère ${ }^{36}$. Mathilde Hautereau-Boutonnet et Ève Truilhé-Marengo ${ }^{37}$ consacrent leur analyse à la biodiversité. Ces deux textes reflètent deux orientations méthodologiques opposées. Fruit d'un travail préliminaire de recensement des mécanismes normatifs consacrés à la régulation de la pollution atmosphérique, l'article de Marion Bary et Marie-Hélène Hubert illustre les difficultés suscitées par l'insertion, dans les normes juridiques, d'éléments d'évaluation économique des biens environnementaux qui mènent à une recomposition du droit, encore problématique en pratique. Le second article rend compte d'une réflexion de long terme sur l'un des objets mis au service de la biodiversité : la notion de valeur. Les auteures mettent l'accent sur la construction progressive d'un cadre conceptuel et méthodologique propre à l'appréhension interdisciplinaire de cette notion, travail qui a abouti à un séminaire interdisciplinaire immersif réussi.

On le perçoit rapidement à la lecture des articles composant ce dossier, l'interdisciplinarité, fut-elle cantonnée, comme en l'espèce, par une double contrainte - l'étude d'objets et pratiques scientifiques et techniques et l'accent mis sur les dimensions normatives de cette étude - se présente sous des formes extrêmement variées. S'il ne devait être qu'un point commun à l'ensemble de ces productions, il faut le chercher, nous semble-t-il, dans la nécessaire humilité que sa pratique suscite chez celles et ceux qui l'expérimentent. L'intelligence mutuelle à laquelle Leibniz fait référence dans la citation liminaire de notre dossier suppose que chacun abandonne, au profit d'une recherche interdisciplinaire constructive, la position d'autorité à laquelle il est parfois tentant de se laisser aller et une bonne partie des réflexes disciplinaires qu'il n'est plus besoin de justifier aux yeux de ses pairs en temps normal. Les déplacements théoriques et épistémologiques qui en découlent, pour ceux qui se lancent dans l'aventure, témoignent, croyons-nous, de la réussite de leur entreprise.

$34 \mathrm{Ou}$, pour reprendre une distinction proposée par Roscoe Pound et familière aux chercheurs en sociologie du droit, "Law in the books " mais aussi "Law in action », sur cette distinction, V. notamment S. Silbey, «After Legal Consciousness », Annu. Rev. Law Soc. Sci., n 1, 2005, p. 323368.

35 L. DANGUY et N. ForTAnÉ, «Les frontières floues de l'expertise scientifique. Le cas de la normalisation du méedicament vétérinaire », p. 103.

36 M. BARY et M.-H. HuBERT, « Instruments juridiques et économiques de régulation de la pollution de l'air et de l'atmosphère », p. 123.

37 M. HAutereau-Boutonnet et E. TruilhÉ-MARENGO, « Recherche interdisciplinaire sur les valeurs de la biodiversité. Acte 1 », p. 145. 